ARTICLE

DOI: $10.1038 / s 41467-017-00572-x$

\title{
A potent series targeting the malarial cGMP- dependent protein kinase clears infection and blocks transmission
}

David A. Baker (1) ', Lindsay B. Stewart ${ }^{1}$, Jonathan M. Large ${ }^{2}$, Paul W. Bowyer ${ }^{1}$, Keith H. Ansell ${ }^{2}$, María B. Jiménez-Díaz ${ }^{3}$, Majida El Bakkouri1 ${ }^{4} 5$, Kristian Birchall ${ }^{2}$, Koen J. Dechering ${ }^{6}$, Nathalie S. Bouloc ${ }^{2}$, Peter J. Coombs², David Whalley², Denise J. Harding², Ela Smiljanic-Hurley², Mary C. Wheldon², Eloise M. Walker ${ }^{1}$, Johannes T. Dessens ${ }^{1}$, María José Lafuente ${ }^{3}$, Laura M. Sanz ${ }^{3}$, Francisco-Javier Gamo ${ }^{3}$, Santiago B. Ferrer ${ }^{3}$, Raymond Hui ${ }^{4,5}$, Teun Bousema ${ }^{7}$, Iñigo Angulo-Barturén ${ }^{3}$, Andy T. Merritt ${ }^{2}$, Simon L. Croft ${ }^{1}$, Winston E. Gutteridge ${ }^{1}$, Catherine A. Kettleborough ${ }^{2} \&$ Simon A. Osborne ${ }^{2}$

To combat drug resistance, new chemical entities are urgently required for use in next generation anti-malarial combinations. We report here the results of a medicinal chemistry programme focused on an imidazopyridine series targeting the Plasmodium falciparum cyclic GMP-dependent protein kinase (PfPKG). The most potent compound (ML10) has an $\mathrm{IC}_{50}$ of $160 \mathrm{pM}$ in a PfPKG kinase assay and inhibits $P$. falciparum blood stage proliferation in vitro with an $\mathrm{EC}_{50}$ of $2.1 \mathrm{nM}$. Oral dosing renders blood stage parasitaemia undetectable in vivo using a $P$. falciparum SCID mouse model. The series targets both merozoite egress and erythrocyte invasion, but crucially, also blocks transmission of mature $P$. falciparum gametocytes to Anopheles stephensi mosquitoes. A co-crystal structure of PvPKG bound to ML10, reveals intimate molecular contacts that explain the high levels of potency and selectivity we have measured. The properties of this series warrant consideration for further development to produce an antimalarial drug.

\footnotetext{
${ }^{1}$ Faculty of Infectious and Tropical Diseases, London School of Hygiene \& Tropical Medicine, Keppel Street, London WC1E 7HT, UK. ${ }^{2}$ LifeArc, Accelerator Building, Open Innovation Campus, Stevenage SG1 2FX, UK. ${ }^{3}$ Tres Cantos Medicines Development Campus-Diseases of the Developing World, GlaxoSmithKline, Tres Cantos, 28760 Madrid, Spain. ${ }^{4}$ Structural Genomics Consortium, University of Toronto, MaRS South Tower, 101 College Street, Toronto, ON, Canada M5G 1L7. ${ }^{5}$ Toronto General Hospital Research Institute, 610 University Avenue, Toronto, ON, Canada M5G 2M9. 6 TropIQ Health Sciences, P.O. Box 9101, 6500 HB Nijmegen, The Netherlands. ${ }^{7}$ Department of Medical Microbiology, Radboud University Medical Center, 6525 HP Nijmegen, The Netherlands. Correspondence and requests for materials should be addressed to D.A.B. (email: david.baker@lshtm.ac.uk)
} 
$\mathrm{M}$ alaria, caused by species of the protozoan Plasmodium, remains the most serious parasitic disease in humans. Around 212 million cases of malaria and approximately 429,000 deaths occurred in $2015^{1}$. Prolonged parasite clearance times, and increasing frequencies of treatment failures following treatment of malaria with artemisinin combination therapies are now regularly reported in parts of Southeast Asia ${ }^{2-5}$. This has led to concerns about the emergence and spread of resistance to these relatively new medicines, which are the mainstay globally for the treatment of malaria caused by Plasmodium falciparum ${ }^{6}, 7$. New non-endoperoxide drugs are considered essential for inclusion as components of novel combination treatments, should the artemisinin derivatives eventually fail.

PKG is a cyclic GMP (cGMP)-activated serine/threonine protein kinase that regulates numerous functions in diverse organisms. There is a single $P K G$ gene in malaria parasites and the $P$. falciparum enzyme, encoded by PfPKG (PlasmoDB Gene ID PF3D7_1436600), has previously been shown to have properties distinct from human orthologes ${ }^{8}$. Selective inhibitors of PKG from the related parasite Eimeria, are thought to interact with a small hydrophobic pocket adjoining the ATP-binding site. Access to this pocket is possible due to the presence of a small (threonine) gatekeeper residue in PKG from both Eimeria and Plasmodium (T618 in PfPKG) ${ }^{9}, 10$. All mammalian PKGs and most serine/threonine kinases have a large gatekeeper residue ${ }^{11}$ preventing access to the pocket and making them insensitive to the PKG inhibitors mentioned above and likely explaining the high levels of selectivity observed ${ }^{9,10}$. These PKG inhibitors also block the development of a number of Plasmodium life cycle stages ${ }^{12-18}$ and we have used a chemical genetic approach that exploits the small gatekeeper residue in PfPKG to generate an inhibitorresistant $P$. falciparum transgenic line (T618Q), to demonstrate that this enzyme plays an essential role in blood stage replication in the human host as well as gametogenesis and ookinete motility in the mosquito vector. The compounds block $P$. falciparum blood stage replication by preventing not only schizont rupture and merozoite egress ${ }^{17}$, 19 , but also merozoite invasion of red blood cells ${ }^{12}$. Prevention of egress is in part due to a downstream block in the PKG-dependent function of the protease PfSUB1 ${ }^{20}$ through the inhibition of its release from exonemes, and the subsequent release of microneme proteins required for erythrocyte invasion ${ }^{19}$. Inhibition of PfPKG activity also blocks calcium mobilization that is required for merozoite egress and invasion and which is thought to be mediated through phosphoinositide metabolism ${ }^{13}$. Using a phosphoproteome analysis we recently identified $\sim 70 \mathrm{P}$. falciparum proteins expressed in mature schizonts that are phosphorylated in a PKG-dependent manner. These proteins are involved in a wide range of cellular processes including cell signaling, ion/protein transport, chromatin remodeling, and actomyosin motor function ${ }^{12}$.

Here, we show that our newly synthesized imidazopyridine PKG inhibitors have high potency and selectivity against $P$. falciparum blood stage proliferation in vitro and in vivo, and that they block transmission of gametocytes to Anopheles mosquitoes. We also present co-crystal structures of $P$. vivax $\mathrm{PKG}$ with the inhibitors, which reveal the interactions underpinning the high degree of selectivity we have observed.

\section{Results}

Highly potent PKG inhibitors block blood stage proliferation. Using the Merck imidazopyridine, compound 2 (ML1) (4-[7[(dimethylamino)methyl]-2-(4-fluorophenyl)imidazo[1,2-a]pyridine-3-yl]pyrimidin-2-amine), developed to treat Eimeria infection $^{9}$ as the chemistry starting point, we synthesized new analogs. These were first tested for their ability to inhibit the kinase activity of recombinant PfPKG using a microfluidic mobility shift assay (see Methods). The best $\mathrm{IC}_{50}$ values obtained were $<200 \mathrm{pM}$ (Table 1). Selected compounds were also tested in the kinase assay against a recombinant PfPKG mutant harbouring a T618Q substitution to investigate the importance of the gatekeeper pocket in the inhibitory mechanism. This mutant kinase exhibited a reduced sensitivity to many of the compounds of between 500 and $>100,000$-fold (Table 1 and Supplementary Fig. 1), emphasizing that this rare structural feature of PfPKG, is vital for inhibitory activity.

The ability of the compounds to block P. falciparum asexual blood stage growth in vitro was then determined using a growth inhibition assay. Compounds had $\mathrm{EC}_{50}$ values ranging from $500 \mathrm{nM}$ down to $<5 \mathrm{nM}$ (Table 1). Selected compounds were then further assayed against the P. falciparum PKG gatekeeper mutant line $(\mathrm{T} 618 \mathrm{Q})^{15}$ to determine whether PfPKG is their primary target in the asexual blood stages. This line showed between a 10 to $>1100$-fold reduction in sensitivity to most compounds (Table 1 and Supplementary Fig. 2) confirming on-target activity. Control drugs were tested in parallel to confirm the changed sensitivity of the gatekeeper mutant line was specific. Chloroquine and artemisinin showed equivalent levels of inhibition of both parasite lines, whereas the gatekeeper mutant line is pyrimethamine resistant (as expected) due to the presence of the drug selectable marker included in the plasmid construct used to mediate allelic replacement (Supplementary Table 1). The physical properties of compounds including stability in mouse and human liver microsomes, membrane permeability and lipophilicity were also measured (Supplementary Table 2; Methods) to inform the chemistry programme.

Starting with ML1, and working through several cycles of design and synthesis (see Methods), we generated compounds ML2-10 (Supplementary Fig. 3), which were selected for further analysis (Table 1). ML10 is the most potent with an $\mathrm{IC}_{50}$ of $\sim 160$ $\mathrm{pM}$ against recombinant PfPKG and an $\mathrm{EC}_{50}$ value of $\sim 2 \mathrm{nM}$ against $P$. falciparum blood stage parasite growth. The compound also exhibited the largest difference in sensitivity between the T618Q transgenic line and the WT 3D7 parasites (>1100-fold). This demonstrates an extremely high degree of specificity for PfPKG and indicates that any secondary parasite target is inhibited only at high concentrations of the compound.

High in vivo efficacy was obtained using the $P$. chabaudi model. Compounds ML1, ML4 and ML10 were tested for in vivo efficacy in BALB/c mice infected with $P$. berghei using a Peters 4day test $^{21}$ at a double daily dose of $25 \mathrm{mg} / \mathrm{kg}$ by oral gavage. Reductions in blood stage parasitaemia measured after 4 days treatment compared to untreated controls were between 52.0 and $60.4 \%$ (Fig. 1a). Plasma samples were taken from satellite groups of mice and all three compounds showed good levels of absorption at the 30 min time point, but plasma levels dropped markedly over the next 30 min (Supplementary Fig. 4a) with half-lives of around $1 \mathrm{~h}(\mathrm{ML1}=1.1 \mathrm{~h}, \mathrm{ML} 4=1.0 \mathrm{~h}$, and $\mathrm{ML} 10=0.8 \mathrm{~h})$.

Given that PfPKG has a relatively narrow window of activity spanning schizont rupture and merozoite invasion, and that $P$. berghei develops asynchronously, it was reasoned that a higher in vivo efficacy might be achievable using the synchronous $P$. chabaudi model, which would allow dosing to target the window of PfPKG activity. A single daily oral dose $(50 \mathrm{mg} / \mathrm{kg})$ of either ML1 or ML4 given to groups of five mice just prior to the predicted onset of schizogony, led to a 67.9 and $56.5 \%$ reduction in blood stage parasitaemia, respectively (Fig. 1b). We next compared single and twice daily $50 \mathrm{mg} / \mathrm{kg}$ oral doses of ML10. The twice daily dose ( $3 \mathrm{~h}$ apart) aimed to target the entire period of schizont rupture and invasion with the intention of preventing 
Table 1 Compound potency in kinase and cell-based assays

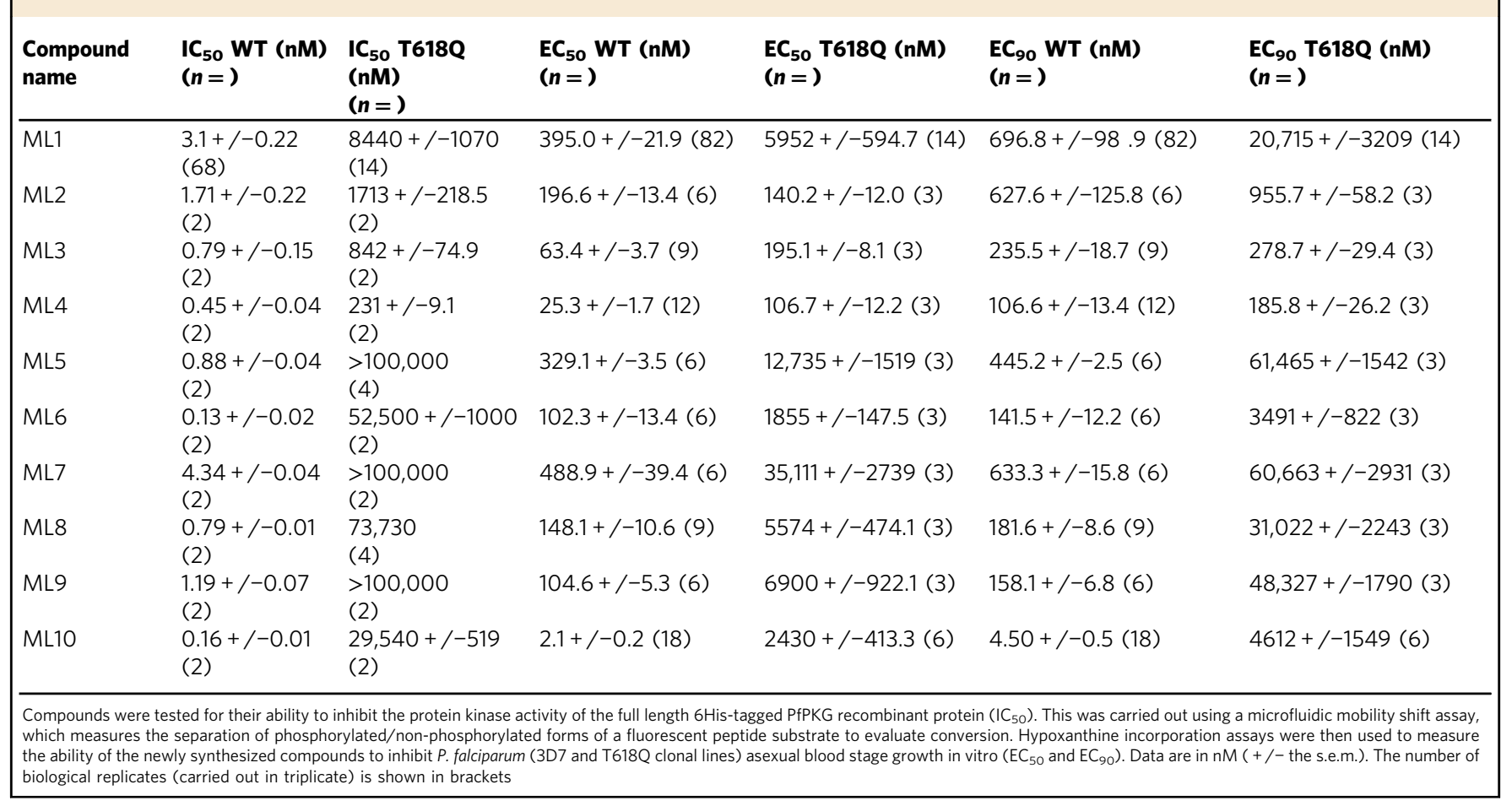

any reinfection. The single dose experiment achieved a $60.9 \%$ reduction in blood stage parasitaemia detected in blood films following 4 days' treatment, whereas the double dose regimen achieved a mean $97.8 \%$ reduction (Fig. 1c). Clearly, the second dose was required to cover the entire period of schizont rupture and re-invasion. There was no overt toxicity in either model. Plasma samples were taken from satellite groups of mice given an oral dose of $25 \mathrm{mg} / \mathrm{kg}$ or $50 \mathrm{mg} / \mathrm{kg}$ ML10. Supplementary Figure $4 \mathrm{~b}$ shows a comparison of the mean plasma levels of ML10 measured at both doses. An almost threefold higher concentration $(10.4 \mu \mathrm{M})$ was measured at the 30 -min time point with the higher dose which was still around $(8.4 \mu \mathrm{M})$ at $60 \mathrm{~min}$. Although this fell to less than $84 \mathrm{nM}$ after $4 \mathrm{~h}$, levels were still well above the $\mathrm{EC}_{90}$ value obtained in the $P$. falciparum growth inhibition assay. The half-life of ML10 in these experiments was $0.8 \mathrm{~h}(25 \mathrm{mg} / \mathrm{kg})$ and $1.1 \mathrm{~h}(50 \mathrm{mg} / \mathrm{kg})$.

Blood stage infection was cleared in a $P$. falciparum mouse model. To further evaluate the efficacy of ML10 it was tested in the GSK $P$. falciparum mouse model (GSKPfalcHuMouse) engrafted with human red blood cells ${ }^{22}$ by administering twice daily doses of 50 or $100 \mathrm{mg} / \mathrm{kg}$ via the oral route to two immunodeficient mice infected with $P$. falciparum 3D70087/N9 for 4 days. Both doses dramatically reduced parasitaemia (Fig. 2a), with levels of ML10 in plasma far above the $\mathrm{EC}_{90}$ in vitro during the $12-\mathrm{h}$ period monitored (Supplementary Fig. 5). With the higher dose, parasitaemia was not detectable at the end of the treatment. The rate of parasite elimination was at least as good as that obtained with mefloquine. The best estimate of potency was $\mathrm{AUC}_{\mathrm{ED} 90}<61.7 \mu \mathrm{g} \mathrm{ml} / \mathrm{h}$, with levels of compound orders of magnitude higher than the $\mathrm{EC}_{90}$ in vitro. After one parasite cycle of exposure, most remaining parasites in peripheral blood were at the late schizont stage (Fig. 2b), suggesting that the process of release of merozoites and invasion of erythrocytes are critical steps of the $P$. falciparum cycle targeted by ML10. The improved efficacy in the GSKPfalcHuMouse model is probably not due to sequence differences between PfPKG and the rodent malaria parasite PKGs, because we previously showed that a transgenic $P$. berghei line in which $P b$ PKG was replaced by PfPKG showed equivalent sensitivity to the chemistry start point $(M L 1)^{23}$. It is likely that the higher dose regimen and the resulting higher plasma concentrations of compounds over time obtained in the SCID experiment were responsible for the significant activity observed. The half-life values for ML10 in these experiments was $8.7 \mathrm{~h}(50 \mathrm{mg} / \mathrm{kg})$ and $17 \mathrm{~h}(100 \mathrm{mg} / \mathrm{kg})$. Importantly, the blood stage of the $P$. falciparum life cycle is $48 \mathrm{~h}$ compared to $24 \mathrm{~h}$ with the rodent malaria parasite species, which likely increases exposure of the sensitive stages to the compound and contributes to the increased efficacy. To investigate the dynamics of parasite killing, an in vitro parasite reduction rate (PRR) assay was carried out $^{24}$. A lag of about $24 \mathrm{~h}$ was observed, during which time the effects of the compounds were reversible following wash-out. However, rapid killing occurred after parasites had been exposed to ML10 for more than $24 \mathrm{~h}$ (Fig. 2c). This killing profile is consistent with PfPKG inhibitors acting at the egress/invasion stage as the starting parasite population in this assay is $>80 \%$ rings. Results are also indicative that inhibition of these cellular processes is lethal and parasites cannot recover even if compound is no longer present. Figure $2 \mathrm{c}$ shows the profile of ML10 compared to those of control antimalarial compounds assayed in the same way.

The series blocks transmission of $\boldsymbol{P}$. falciparum to mosquitoes. We also tested the transmission-blocking effects of this series by pre-incubating mature $P$. falciparum gametocytes (strain NF54) for $24 \mathrm{~h}$ with ML1, ML4, and ML10 and then feeding them to Anopheles stephensi mosquitoes using standard membrane feeding assays (SMFA; ${ }^{25}$ to measure oocyst numbers at the various concentrations). Potent transmission-blocking activity was observed for the new compounds, with $\mathrm{IC}_{50}$ values for reduction of the intensity of infection being 507.3, 61.6 and $41.3 \mathrm{nM}$, respectively, (Fig. 3 and Supplementary Fig. 6). We have 


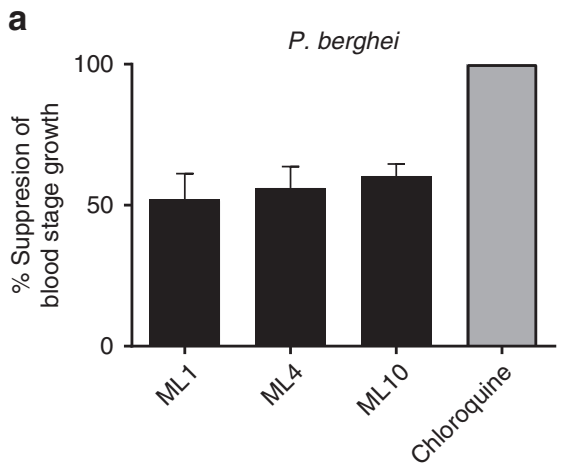

b

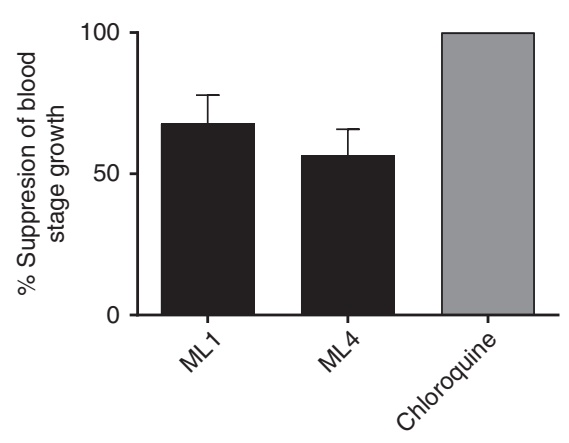

C

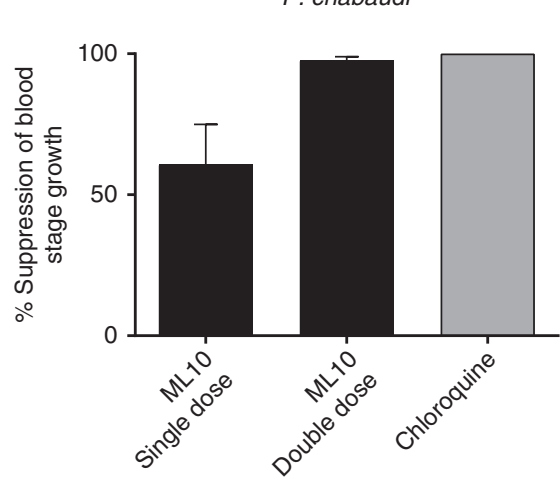

Fig. 1 In vivo efficacy of PKG inhibitors against rodent malaria parasites. a Groups of five female BALB/c mice were infected with $1 \times 10^{7} / \mathrm{ml} P$. berghei (ANKA) blood stage parasites in a Peters 4-day test and were given a twice daily dose $(25 \mathrm{mg} / \mathrm{kg})$ of one of three test compounds by oral gavage. Chloroquine was used as a positive control at a single daily oral dose of $10 \mathrm{mg} / \mathrm{kg}$. b Groups of five female BALB/c mice were infected with $1 \times 10^{7}$ P. chabaudi (AS) blood stage parasites and were given a single oral dose $(50 \mathrm{mg} / \mathrm{kg}$ ) of either ML1 or ML4 by oral gavage just prior to the predicted onset of schizogony. Chloroquine was used as a positive control at a single daily oral dose of $10 \mathrm{mg} / \mathrm{kg}$. c Groups of five BALB/c mice were infected with $1 \times 10^{7}$ P. chabaudi (AS) blood stage parasites and were given either a single or twice daily oral dose $(50 \mathrm{mg} / \mathrm{kg})$ of ML10 by oral gavage. The first dose was given to both groups of mice just prior to the predicted onset of schizogony and in one group this was followed $3 \mathrm{~h}$ later when schizogony was predicted to have been completed. The data are from single experiments each performed on a group of five mice. Error bars show the s.e.m

previously shown that PKG is essential for gametocyte activation and transformation into gametes ${ }^{15}$, and propose that inhibition of these events underlies the observed transmission-blocking activity. To confirm the mechanism of action of ML10 in gametocytes, we compared the ability of ML1 and ML10 to block rounding up of $P$. falciparum wild-type (3D7) and gatekeeper mutant (T618Q) stage $\mathrm{V}$ gametocytes. Both compounds blocked rounding up of wild-type gametocytes, but the majority of T618Q gametocytes were able to round up in the presence of both PKG inhibitors showing that they are insensitive to the compounds and thereby confirming that the primary target of ML10 (and ML1 as previously shown ${ }^{15}$ ) is PKG in gametocytes as well as in blood stages (Supplementary Fig. 7).

ML10 has little activity against human kinases and cell lines. To assess the selectivity of ML10, it was tested against a panel of 80 human protein kinases (representing all the main families and including 14 small gatekeeper kinases) and it showed a clean profile with only low levels of inhibitory activity when tested at $100 \mathrm{nM}\left(>600 \times \mathrm{IC}_{50}\right.$ against recombinant PfPKG) (Supplementary Fig. 8). Although achieved through an independent experimental approach, this result is consistent with the observed $>1100$-fold difference in sensitivity between wild-type and gatekeeper mutant parasites reflecting the exquisite specificity of this compound for PfPKG. Of the panel of 80 human kinases, the highest level of inhibition was obtained with human MLK3 (40\% at $100 \mathrm{nM}$ ). MLK3 has a very low overall similarity to PfPKG with their kinase domains having only 22\% identity. Human MLK3 has a large hydrophobic residue (methionine) in the gatekeeper position, which would prevent access of the inhibitor to the gatekeeper pocket and the $\mathrm{IC}_{50}$ for ML10 with MLK3 is $>600$-fold higher than with PfPKG. The three MLK isoforms most similar to MLK3 are MLK1, MLK2 and MLK4 and these also have bulky hydrophobic gatekeeper residues. Compounds ML1-10 were tested in cytotoxicity assays using HepG2 cells (derived from liver hepatocellular carcinoma). ML10 and most of those tested had $\mathrm{EC}_{50}$ values higher than the maximum dose tested (Supplementary Table 2). To provide additional safety data for ML10, it was tested against three additional human cell lines at concentrations between 0.001-10 $\mu \mathrm{M}(n=6$; CXR Biosciences Ltd; Supplementary Fig. 9) derived from lung carcinoma (A549), colorectal adenocarcinoma (HT-29), and breast adenocarcinoma (MCF7). For all three lines the $\mathrm{EC}_{50}$ values for ML10 were higher than the highest concentration used $(10 \mu \mathrm{M})$ confirming a very high selectivity window ( $>4500$ fold) for this compound.

To investigate whether resistance is readily generated to this class of inhibitor using prolonged exposure of parasites to sublethal doses, we followed a published protocol ${ }^{26}$. No resistant parasites were selected, suggesting that substitutions in the compound-binding domain (or at a locus responsible for an alternative resistance mechanism) are not readily selected under these conditions. Drug resistant parasites were, however, readily selected in control cultures treated with atovaquone, but test cultures treated with the PKG inhibitor remained negative for the duration of the experiment (Methods; Supplementary Fig. 10). 
a

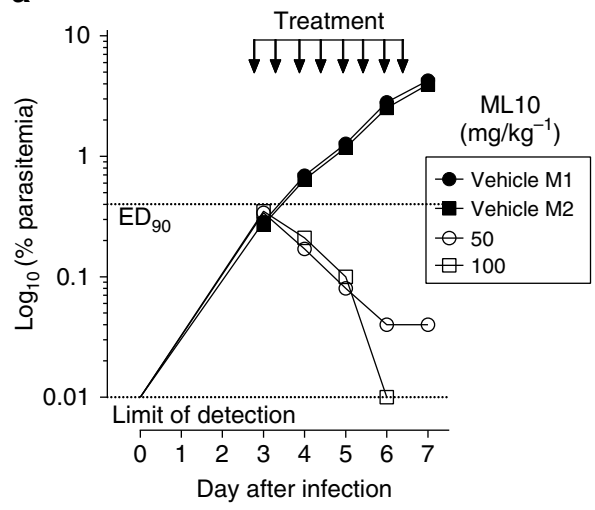

C

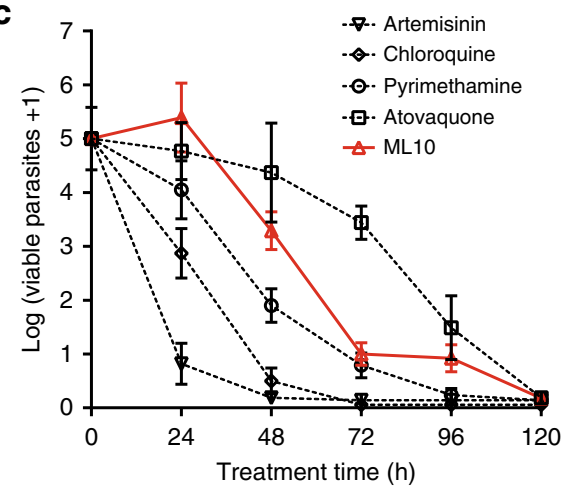

b

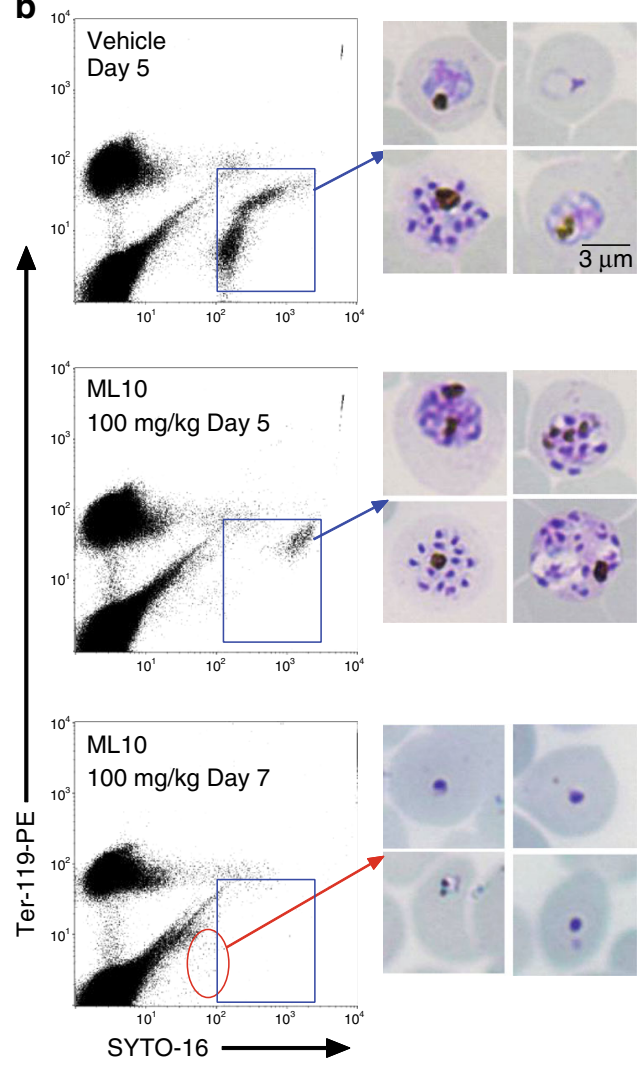

Fig. 2 Efficacy of ML10 against P. falciparum in the GSK PfalcHuMouse model and determination of in vitro killing dynamics. a Two mice were treated with vehicle and another two mice with either 50 or $100 \mathrm{mg} / \mathrm{kg}$ of ML10 to test proof of concept of efficacy in vivo. Parasitemia is shown over time in individual mice during the efficacy assay. The dotted horizontal line indicates $90 \%$ reduction in parasitaemia compared to vehicle-treated animals. Each symbol represents an individual mouse. $\mathbf{b}$ Microscopic and flow cytometric analysis of $P$. falciparum present in the peripheral blood of mice treated with vehicle or ML10. Samples taken after one $(48 \mathrm{~h})$ and two cycles $(96 \mathrm{~h})$ of exposure to the test compound were further analyzed. Flow cytometry dot plots from samples of peripheral blood show P. falciparum-infected human erythrocytes (blue rectangle). Images in the right-hand panels show Giemsa-stained blood stage parasites. Blood films from control untreated animals show normal staining and appearance. The parasites in ML10-treated animals show a relative enrichment in late schizonts at Day 5, whereas most cells remaining in peripheral blood at Day 7 are pyknotic (red circle). Scale bar, $3 \mu \mathrm{m}$. c The in vitro parasite reduction rate $(P R R)$ assay was used to determine the onset of action and rate of killing as previously described ${ }^{24}$. $P$. falciparum was exposed to ML10 at a concentration corresponding to $10 \times E_{50}$. The number of viable parasites at each time point was determined as described ${ }^{24}$. Four independent serial dilutions were tested with each sample to correct for experimental variation; error bars show the standard deviation. Previous results reported on standard antimalarials tested at $10 \times \mathrm{EC}_{50}$ using the same conditions are shown for comparison ${ }^{24}$

Crystal structures give insight into the selectivity observed. To seek insights into the interactions between Plasmodium PKG and the new inhibitors, we expressed, purified and crystallized recombinant protein samples from $\mathrm{PfPKG}$ and $P$. vivax $\mathrm{PKG}$ (PvPKG), but only obtained diffracting crystals from the latter with ML1 (PDB: 5FET) and ML10 (PDB: 5EZR). The two orthologe are $92 \%$ identical in sequence over the full length sequences. The kinase domains of the apo structures of the two orthologe, when their atomic positions are aligned (Supplementary Fig. 11), deviate from each other by a negligible root-meansquare distance (RMSD) of $0.3 \AA$. Clearly, PvPKG is a suitable structural surrogate of PfPKG. We obtained high resolution cocrystals of PvPKG with ML1 and ML10, respectively. The data collected for the resulting co-structures (PDB: 5FET with ML1; 5EZR with ML10) showed clear electron density for each compound. Alignment of their kinase domains with those from the PvPKG apo structure (PDB: 5DYL) and a co-structure with adenylyl imidodiphosphate (AMPPNP), a non-hydrolysable analog of ATP (PDB: 5DZC) resulted in RMSD values lower than $0.3 \AA$, showing that ligand binding changed the conformation of the protein negligibly (Supplementary Fig. 12). We also note that all ligands formed complexes with the cGMP-free and inactive state of PvPKG. To date, we have not succeeded in our attempts to crystallize full length PKG from either Plasmodium species with cGMP bound, with or without inhibitors or other ligands in the catalytic domain. Comparison of the inhibitorbound structures reveals two key contributors to potency shared by both compounds. First, hydrogen bonds between the aminopyrimidine and the backbone of V614 (V621 in PfPKG; Fig. 4a) in both PvPKG structures form the quintessential hinge interaction previously observed with many ATP-mimicking kinase inhibitors. Second, the fluorophenyl group occupies the hydrophobic pocket next to the relatively small gatekeeper (T611; T618 in PfPKG; Fig. 4b). The latter interaction also confers selectivity, as this pocket would be blocked by the sidechain of a larger gatekeeper residue, such as that found in the T618Q mutant and the majority of S/T kinases (in both humans and parasites). The pocket extends beyond the gatekeeper (Fig. 4b), with the additional cavity unexploited by ML1 but filled by the sulfonamide group in ML10 engaging in hydrogen bonds with D675 (D682 in PfPKG) and F676 (F683 in PfPKG) of the DFG triad (Fig. 4a). Finally, both compounds engage in hydrophobic interactions with a network of residues from both lobes and the hinge of the kinase domain (Fig. 4c and Supplementary Fig. 13). Notably, the 


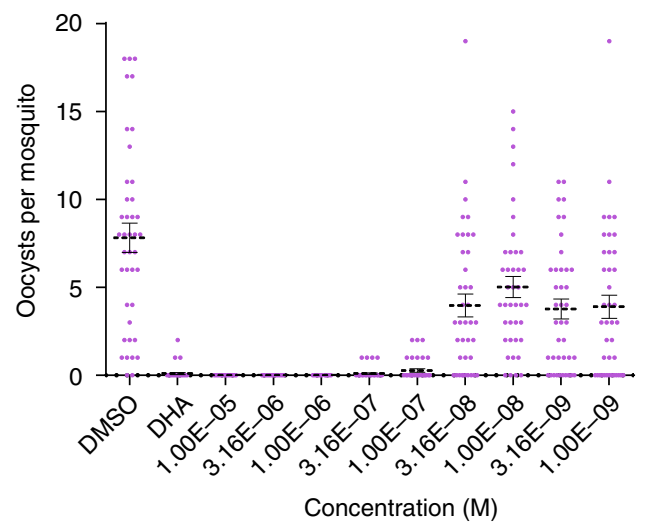

Fig. 3 Transmission-blocking activity of ML10. P. falciparum (NF54) gametocytes were incubated for $24 \mathrm{~h}$ with nine different concentrations of ML10 and were each fed to a separate cage of 30-40 Anopheles stephensi mosquitoes using a SMFA. Up to 20 surviving mosquitoes were dissected on day 7 post-feed and oocyst numbers assessed by microscopy. This experiment was performed twice and the figure shows the combined data for oocyst intensity in each mosquito as a function of the compound concentration for the replicate feeders. The left segment of the $x$-axis shows the oocyst intensities in the vehicle $(0.1 \%$ DMSO) controls. The positive control was $10 \mu \mathrm{M}$ dihydroartemisinin (DHA). Error bars show the s.e.m

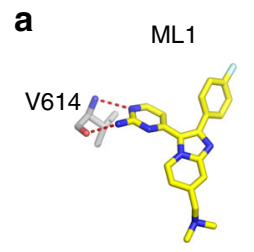

b

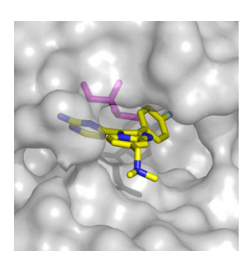

C

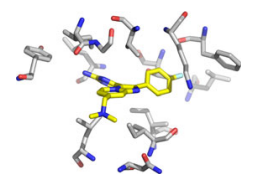

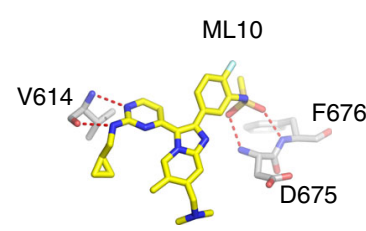
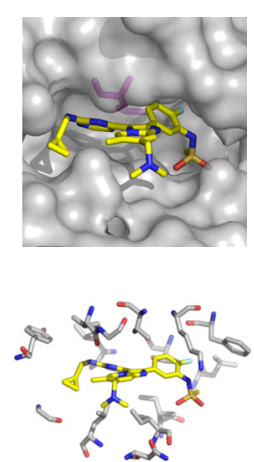

Fig. 4 Side-by-side comparison with key features in the PvPKG/ML1 and PvPKG/ML10 co-structures. a Hydrogen bonds provide key interactions: At the hinge of the kinase domain, V614 forms hydrogen bonds with the amino-pyrimidine group of both ML1 (left) and ML10 (right). In addition, ML10 extends a sulfonamide moiety to form hydrogen bonds with D675 and F676, resulting in significantly greater inhibitory potency than ML1.

b Gatekeeper confers specificity: Both ML1 (left) and ML10 (right) extend a fluorophenyl group to occupy a hydrophobic pocket adjacent to T611 (magenta). This explains in part the specificity of the inhibitors as a residue with a longer side-chain may potentially clash with this functional group. c Hydrophobic network enhances inhibitory potency: Plasmodium PKG also interacts with both ML1 (left) and ML10 using a network of hydrophobic residues (see Supplementary Fig. 13 for the same figure with amino-acid labels)

\begin{tabular}{|c|c|c|}
\hline & PvPKG-ML1 & PvPKG-ML10 \\
\hline $\begin{array}{l}\text { PDB } \\
\text { Data collection }\end{array}$ & 5FET & $5 \mathrm{EZR}$ \\
\hline $\begin{array}{l}\text { Space group } \\
\text { Cell dimensions }\end{array}$ & $\mathrm{C} 2$ & $\mathrm{C} 2$ \\
\hline $\begin{array}{l}a, b, c(\AA) \\
\quad \alpha, \beta, \gamma\left({ }^{\circ}\right) \\
\text { Resolution } \\
R_{\text {sym }} \\
l / \sigma l \\
\text { Completeness (\%) } \\
\text { Redundancy }\end{array}$ & $\begin{array}{l}190.5,117.2,67.4 \\
90.0,94.3,90.0 \\
45.00-3.07(3.18) \\
0.12(0.85) \\
15.3(2.2) \\
99.4(99.2) \\
4.2(4.3)\end{array}$ & $\begin{array}{l}191.1,118.0,68.2 \\
90.0,95.2,90.0 \\
50.00-2.50(2.54) \\
0.08(0.68) \\
19.2(1.5) \\
97.7(81.9) \\
4.1(3.9)\end{array}$ \\
\hline \multicolumn{3}{|l|}{ Refinement } \\
\hline Resolution $(\AA)$ & $44-3.07$ & $50-2.50$ \\
\hline No. of reflections & 28,885 & 50,525 \\
\hline $\begin{array}{l}R_{\text {work }} / R_{\text {free }} \\
\text { No. of atoms }\end{array}$ & $0.23 / 0.25$ & $0.23 / 0.26$ \\
\hline Protein & 6235 & 6203 \\
\hline Compound & 27 & 37 \\
\hline Water & 62 & 69 \\
\hline Average B-factors & 79.4 & 80.7 \\
\hline \multicolumn{3}{|l|}{ RMS deviations } \\
\hline Bond lengths $(\AA)$ & 0.007 & 0.008 \\
\hline Bond angles $\left({ }^{\circ}\right)$ & 0.80 & 1.19 \\
\hline \multicolumn{3}{|l|}{ Ramachandran plot } \\
\hline Favored regions & $97.9 \%$ & $96.1 \%$ \\
\hline Allowed regions & $100 \%$ & $99.9 \%$ \\
\hline
\end{tabular}

methylation of the imidazopyridine core and the cyclopropylmethylene extension from the aminopyrimidine in ML10 enhances this network and consequently increased the potency of inhibition. Crystallography data collection and refinement statistics are shown in Table 2.

\section{Discussion}

Currently there is optimism regarding several new chemical entities undergoing clinical development as antimalarials ${ }^{27-32}$. However, attrition rates in drug development across all therapeutic areas are high. Although the use of protein kinase inhibitors has been a successful strategy for treating a range of human cancers for many years, with 25 approved drugs to date ${ }^{33}$, to our knowledge it has not proved possible to exploit this target class to treat infectious disease. An inhibitor of a $P$. falciparum lipid kinase (PI4K) is showing real promise ${ }^{29}$ and is in clinical development. Although our study has focused on the effects of the new PKG inhibitors on blood stages and sexual stages, it has been reported previously that PKG has roles in liver stages ${ }^{34}$ and sporozoites $^{18}$ and so it would be worth exploring the effects of the new compounds on these additional stages to prevent the initiation of a blood stage infection. Our results suggest that ML10 or an analog with further optimized pharmacokinetic properties, targeting the protein kinase PfPKG, might have significant efficacy in terms of curing malaria in patients as well as blocking transmission within the population in the context of malaria elimination programmes ${ }^{35}$. Selectivity to avoid toxicity and side effects is a major challenge in antimalarial chemotherapy, especially when targeting protein kinases. However, our results with the malaria parasite PKG with its small gatekeeper residue conferring a rare structural feature, have demonstrated that a highly selective inhibitor can be generated with sufficient potency to clear P. falciparum infection in vivo. Altogether our results suggest that PKG inhibitors should be considered for 
development as a component of a future antimalarial combination treatment.

\begin{abstract}
Methods
Medicinal chemistry strategy summary. The chemistry program started with the Merck imidazopyridine, Compound 2, here referred to as ML1 (signifying their generation through a partnership between MRC Technology (now LifeArc) and the London School of Hygiene and Tropical Medicine). Removing the dimethylbenzylamine and attaching a basic amine to the aminopyrimidine via a phenyl spacer (ML2) improved both cell potency and microsomal stability, but drastically reduced permeability. Reintroduction of the dimethylamine (ML3) further improved potency and restored permeability. A further boost to potency came with the addition of a methyl (ML4) vicinal to the dimethylbenzylamine. Going back to ML1, changing the 4-fluorophenyl to 3-methylsulphonylphenyl (ML5) gave similar potency and better microsomal stability to (ML1) but poorer permeability. Addition of a cyclopropylmethylene group to the aminopyrimidine (ML6) improved both potency and permeability with a slight drop off in microsomal stability. Moving to the 3-methylsulphonamidophenyl (ML7), both potency and permeability were poorer than (ML1), though reintroduction of the 4-fluoro group (ML8) boosted potency but not permeability. Addition of the vicinal methyl (ML9) again gave a slight improvement in potency and permeability and the addition of the cyclopropylmethylene group (ML10) gave a compound with highly potent antimalarial activity. Analytical data are shown in Supplementary Note 1.
\end{abstract}

\section{Expression and purification of recombinant PKG. Full length $P f P K G$ (NCBI} accession code XP_001348520) with native codon usage was cloned into the pTrcHisC plasmid (Life Technologies) that includes an $\mathrm{N}$-terminal His-tag as described previously ${ }^{8}$. PfPKG with threonine 618 replaced with a glutamine (PfPKG T618Q) was cloned into the same plasmid as described previously ${ }^{15}$. Recombinant proteins were generated and purified using a protocol based on that described previously ${ }^{8}$. Briefly, freshly transformed E. coli Rosetta ${ }^{\mathrm{TM}}$ 2(DE3) pLysS (Novagen; Cat. No. 71403) were used for expression of recombinant PfPKG. $500 \mathrm{ml}$ cultures in LB Rich Broth (containing $50 \mu \mathrm{g} / \mathrm{ml}$ carbenicillin and $34 \mu \mathrm{g} / \mathrm{ml}$ chloramphenicol) were grown in a shaking incubator at $37^{\circ} \mathrm{C}$ until reaching an optical density (O.D.) of $0.6-0.7$. The temperature was reduced to $16^{\circ} \mathrm{C}$ before induction of expression with $1 \mathrm{mM}$ IPTG. Incubation at $16^{\circ} \mathrm{C}$ was continued overnight.

The cultures were harvested by centrifugation at $4000 \times g$ at $4^{\circ} \mathrm{C}$ for $30 \mathrm{~min}$, the supernatant removed and the pellet stored at $-80^{\circ} \mathrm{C}$ for in excess of $1 \mathrm{~h}$. The PKGs were purified via the histidine tag on HiTrap TALON (cobalt) columns (GE Healthcare) connected to an AKTA-FPLC as per the manufacturer's instruction. Fractions were analyzed by SDS-PAGE and the main peak concentrated on $10 \mathrm{kDa}$ MWCO concentrators (Amicon). Purified proteins were stored in $50 \%$ glycerol at $-80{ }^{\circ} \mathrm{C}$ in single use aliquots. The final buffer composition of the purified product was: $50 \mathrm{mM}$ Tris/HCl pH 7.5, $0.1 \mathrm{mM}$ EGTA, $150 \mathrm{mM} \mathrm{NaCl}, 0.1 \%$

$\beta$-mercaptoethanol, $50 \%$ glycerol, $0.03 \%$ Brij- $35,1 \mathrm{mM}$ benzamidine and $0.2 \mathrm{mM}$ PMSF. PfPKG protein preparation was outsourced at the Division of Signal Transduction Therapy, School of Life Sciences, University of Dundee.

Microfluidic assay for recombinant PfPKG. $\mathrm{IC}_{50}$ values were determined for test compounds using a microfluidic mobility shift assay. Briefly, compounds were prepared over a 10 -well $1 / 2 \log$ dilution series in dimethyl sulfoxide (DMSO) in duplicate in $50 \mu \mathrm{l}$ volumes using 384-well polypropylene U-bottomed plates (Thermo Scientific, UK). The plates contained positive/no inhibitor (DMSO only) and negative (no enzyme) controls in columns 1,2 , and 23,24 . The reaction mix for each well consisted of $20 \mu \mathrm{l}$ of enzyme/peptide mix (1.25 nM PfPKG, $1.5 \mu \mathrm{M}$ FAM-labeled PKAtide [FAM-GRTGRRNSI-NH2, Cambridge Bioscience, UK] in PfPKG assay buffer [ $25 \mathrm{mM}$ HEPES (pH 7.4), $20 \mathrm{mM} \beta$-glycerophosphate, $2 \mathrm{mM}$ DTT, $10 \mu \mathrm{M}$ cGMP, $0.01 \%(\mathrm{w} / \mathrm{v})$ BSA, $0.01 \%(\mathrm{v} / \mathrm{v})$ Triton X-100]) plus $5 \mu \mathrm{l}$ of compound. Samples were pre-incubated at room temperature for $30 \mathrm{~min}$ and reactions were initiated by addition of $25 \mu \mathrm{l}$ ATP $\operatorname{mix}\left(10 \mathrm{mM} \mathrm{MgCl}_{2}\right.$ and ATP, at $\mathrm{KM}$ of the enzyme under test [20 $\mu \mathrm{M}$ PfPKG and $90 \mu \mathrm{M}$ PfPKG T618Q], in water). Positive controls were complete reaction mixtures with $10 \%$ DMSO and negative controls were reaction mixtures with $10 \%$ DMSO but lacking enzyme. Reactions were allowed to proceed for $30 \mathrm{mins}$ at room temperature, corresponding to conversion of approximately $10 \%$ of the substrate in the DMSO controls. Reactions were terminated by addition of $50 \mu \mathrm{l}$ stop solution ( $25 \mathrm{mM}$ EDTA in water). Samples were analyzed by electrophoretic separation of substrate and product peaks and fluorescence detection using a Caliper Lab Chip EZ reader. (Perkin Elmer, Waltham MA) with $0.2 \mathrm{~s}$ sip time, downstream voltage $500 \mathrm{~V}$, upstream voltage $1950 \mathrm{~V}$ and pressure 0.5 to $1.5 \mathrm{psi}$. Substrate and product peak heights were measured and the ratio of the product peak height divided by the sum of the product and substrate peaks were determined using EZ reader software (version 3.0.265.0) to obtain percentage conversion $(P)$ values. $P$-values were normalized to percentage activity relative to positive and negative controls were $\%$ activity $=$ $100 \times\left(P-P_{\text {neg ctrls }}\right) /\left(P_{\text {pos ctrls }}-P_{\text {neg ctrls }}\right)$ and fitted to obtain $\mathrm{IC}_{50}$ values using a 4-parameter logistical fit (XL-fit, IDBS, Guildford UK). Liquid handling stages were conducted on a Biomek robotic liquid handler (Beckman Coulter).
Plasmodium falciparum growth inhibition assays. We determined that a $72 \mathrm{~h}$ P. falciparum (clone 3D7A; obtained from Lisa Ranford Cartwright, University of Edinburgh) growth inhibition assay was necessary for full inhibition to manifest itself and that this is due, most likely to a schizont-specific action rather than a delayed death phenotype as observed for some antibiotic macrocycles. Hypoxanthine incorporation assays ${ }^{36}$ were performed on P. falciparum asexual blood stage parasites (wild-type $3 \mathrm{D} 7$ or $\mathrm{T} 618 \mathrm{Q}$ gatekeeper mutant) to determine $\mathrm{EC}_{50}$ values for test compounds. Mixed staged parasites (primarily rings) were added to 96 well plates containing compounds at concentrations of $50 \mu \mathrm{M}-0.3 \mathrm{nM} .\left[{ }^{3} \mathrm{H}\right]-$ Hypoxanthine was added after $48 \mathrm{~h}$ to a final concentration of $0.2 \mu \mathrm{Ci}$. The radioactivity of the labeled hypoxanthine incorporated into the parasite nucleic acids over $24 \mathrm{~h}$ was determined relative to untreated controls using a Wallac 1450 Microbeta scintillation counter (Perkin-Elmer). Assays were carried out in triplicate at least twice.

Peters 4-day test using the asynchronous $\boldsymbol{P}$. berghei ANKA model. PKG inhibitors were tested for in vivo efficacy using randomized female $B A L B / c$ mice (aged 6-8 weeks; Charles River Laboratories, Harlow, UK) infected intravenously with $1 \times 10^{7} / \mathrm{ml}$ P. berghei (ANKA; from stocks held at LSHTM) blood stage parasites in a standard 4 -day test ${ }^{21}$. Animals in the test group were dosed once or twice daily for 4 consecutive days beginning on the day of infection (i.e., $D 0$ to $D+$ 3) at 25 or $50 \mathrm{mg} / \mathrm{kg}$ via the oral route (po). Animals in control groups were dosed once daily with chloroquine at $10 \mathrm{mg} / \mathrm{kg} / \mathrm{po}$ or daily with pyrimethamine at $0.3 \mathrm{mg} /$ $\mathrm{kg} / \mathrm{po}$ for 4 consecutive days. Reductions in blood stage parasitaemia were measured after 4 days' treatment.

Synchronous $\boldsymbol{P}$. chabaudi model. Similar experiments were performed using P. chabaudi AS (from David Walliker, University of Edinburgh) with the following modifications. To allow dosing to be carried out more conveniently during the day, reversal of the light/dark exposure of mice for 2 weeks prior to the experiment was used to switch schizogony from midnight to noon each day. Mice were dosed at different time points in an attempt to have maximal coverage at the point of schizogony. All animal work protocols carried out at LSHTM were approved and licensed by the United Kingdom Home Office as governed by law under the Animals (Scientific Procedures) Act of 1986, in strict accordance with the Code of Practice Part 1 for the housing and care of animals (21 March 2005), available at http://www.homeoffice.gov.uk/science-research/animal-research/

Efficacy against $\boldsymbol{P}$. falciparum in vivo. The efficacy of ML10 against the $P$. falciparum 3D7 line was tested in the GSK $P$. falciparum humanized mouse model (GSKPfalcHuMouse) $^{22}$. The uncloned P. falciparum 3D7 line was kindly donated by Drs. E. Dei-Cas and L. Delhaes from Institut Pasteur (Lille, France) and was adapted to grow in peripheral blood of engrafted NODscid/ $\beta 2 \mathrm{~m}-/-\mathrm{mice}^{37}$. One of the isolates obtained ( $\left.P f 3 D 7^{0087 / N 9}\right)$ was expanded in vivo and established as a reference strain for animal mouse model development.

Briefly, age-matched (8-10-weeks old) female immunodeficient NOD-scid IL2R $\gamma$ c-null mice (The Jackson Laboratory, Bar Harbor, ME) were engrafted with human erythrocytes (Red Cross Transfusion Blood Bank in Madrid, Spain) by intraperitoneal daily injection with $1 \mathrm{ml}$ of a $50 \%$ hematocrit erythrocyte suspension (RPMI 1640 medium, 25\% (vol/vol) decomplemented human serum, $3.1 \mathrm{mM}$ hypoxanthine) throughout the experiment. The mice were infected with $2 \times 10^{7}$ P. falciparum Pf3D $7^{0087 / N 9}$-infected erythrocytes (Day 0) at $\sim 40 \%$ chimerism in peripheral blood. The drug treatment was given to mice on Day 3 after infection every $12 \mathrm{~h}$ for 4 consecutive days by oral gavage in a volume of administration of $10 \mathrm{ml} / \mathrm{kg}$ bodyweight, at a dose of 50 or $100 \mathrm{mg} / \mathrm{kg}$ bodyweight. ML10 was prepared in $90 \% \mathrm{ddH}_{2} \mathrm{O}, 7 \%$ Tween $80,3 \%$ ethanol. Parasitemia was measured by flow cytometry in samples of peripheral blood stained with the fluorescent nucleic acid dye SYTO-16 (Molecular Probes, Cat. No.: S-7578) at a concentration of $5 \mu \mathrm{M}$ and anti-murine erythrocyte TER119 monoclonal antibody $(10 \mu \mathrm{g} / \mathrm{ml}$; Beckton Dickinson) in serial $2 \mu \mathrm{L}$ blood samples taken every $24 \mathrm{~h}$ until assay completion as described ${ }^{22}$. AUC $\mathrm{ED}_{90}$ is the average daily exposure of the compound in whole blood that reduces parasitemia at day 7 of the in vivo assay by $90 \%$ with respect to parasitemia in vehicle-treated mice.

The plasma levels of ML10 in mice from the efficacy experiment were measured in serial samples of peripheral blood $(25 \mu \mathrm{l})$ taken by tail puncture at $0.25,0.5,2,4$, 6 , and $12 \mathrm{~h}$ after the first administration. The blood samples were immediately lysed by mixing with $25 \mu \mathrm{l}$ of distilled water, frozen on dry ice and stored at $-80^{\circ} \mathrm{C}$ until analysis. The compounds were extracted from $10 \mu \mathrm{l}$ of each lysate by liquid-liquid extraction in the MultiScreen Solvinert $0.45 \mu \mathrm{m}$ Hydrophobic PTFE 96- well plate system (Millipore) and stored frozen at $-80^{\circ} \mathrm{C}$ until analysis by $\mathrm{LC} /$ MS/MS in API4000 (AB Sciex, Framingham, MA). The compound concentration vs. time data were analyzed by non-compartmental analysis using Phoenix ${ }^{\circledR}$ Version 6.3 (Pharsight Corporation, Mountain View, CA, USA). Additional statistical analysis was performed with GraphPad Prism ${ }^{\circledR}$ Version $6.02(\mathrm{GraphPad}$ Software Inc, San Diego CA, USA).

All the experiments were approved by the DDW Ethical Committee on Animal Research, performed at the DDW Laboratory Animal Science facilities accredited by AAALAC, and conducted in accordance with European Directive 86/609/EEC and the GSK Policy on the Care, Welfare and Treatment of Animals. The human 
biological samples were sourced ethically and their research use was in accord with the terms of the informed consent. Erythrocyte concentrates from malaria-negative donors were provided by Biobancos de Castilla y Leon, Barcelona and Centro de Transfusiones de Madrid and the Red Cross Transfusion Blood Bank in Madrid, Spain. Research was conducted according to POL-GSKF-410 and was in accord with the terms of the informed consent of each donor.

In vitro parasite reduction ratio. In vitro PRR testing was conducted at GlaxoSmithKline (Tres Cantos, Madrid, Spain) as previously described ${ }^{24}$. The assay used the limiting dilution technique to quantify the number of parasites that remained viable after drug treatment. P. falciparum strain 3D7A (Malaria Research and Reference Reagent Resource Center (MR4), BEI Resources; Cat. No. MRA102) was treated with a drug concentration corresponding to $10 \times \mathrm{IC}_{50}$. Conditions of parasites exposed to treatment were identical to those used at GSK in the $\mathrm{IC}_{50}$ determination ( $2 \%$ hematocrit, $0.5 \%$ parasitemia). Parasites were treated for $120 \mathrm{~h}$. Drug in culture medium was renewed daily over the entire treatment period. Parasite samples were collected from the treated culture every $24 \mathrm{~h}(24,48,72,96$, and $120 \mathrm{~h}$ time points); drug was washed out of the sample, and parasites were cultured drug-free in 96-well plates by adding fresh erythrocytes and culture medium. To quantify the number of viable parasites after treatment, threefold serial dilutions were used with the above-mentioned samples after removing the drug. Four independent serial dilutions were performed with each sample to correct experimental variations. The number of viable parasites was determined after 21 and 28 days by counting the number of wells with growth using $\left[{ }^{3} \mathrm{H}\right]$-hypoxanthine incorporation. The number of viable parasites was back-calculated by using the formula $X^{n-1}$ where $n$ is the number of wells able to render growth and $X$ the dilution factor (when $n=0$, number of viable parasites is estimated as zero).

In vitro selection of resistant parasites. The resistance profiling was carried out according to the assay protocol described previously ${ }^{26}$, which looks for the emergence of resistant mutants against a compound concentration of $3 \times \mathrm{IC}_{50} . P$. falciparum lab isolate Dd2 (from stocks held at LSHTM) was used for this experiment and the control compound used was atovaquone. Parasites were plated in triplicate at $10^{9}$ parasites/well and maintained until parasites were observed microscopically (using Giemsa-stained blood films) or until Day 60 whichever was the soonest. Parasites from the atovaquone plate were discarded at Day 18 and cultures treated with the PKG inhibitor ML1 were parasite negative at Day 60. Gametocytes were present initially but were absent from Day 11.

Standard membrane feeding assays. Mature $P$. falciparum (NF54) gametocytes (14 day culture, $0.3-0.5 \%$ gametocytes, $2 \%$ haematocrit) were obtained from an automated tipper system and incubated with compound for $24 \mathrm{~h}$ as previously described $^{38}$. Anopheles stephensi (Sind-Kasur Nijmegen strain ${ }^{39}$ were reared at $30^{\circ}$ $\mathrm{C}$ and $70-80 \%$ humidity, while exposed to a $12 / 12 \mathrm{~h}$ day/night cycle. On the day of feeding, the gametocyte culture was adjusted to $50 \%$ hematocrit with human red blood cells and human serum and fed to 3-5 day old mosquitoes using a glass membrane mini-feeder system containing $0.35 \mathrm{ml}$ of the $P$. falciparum culture/ compound $\mathrm{mix}^{40}$. Unfed and partially fed mosquitoes were removed after feeding and blood fed females were maintained at $26^{\circ} \mathrm{C}$ and $70-80 \%$ humidity. Up to 20 surviving mosquitoes were collected on day 7 post-infection and oocysts were visualized using a $1 \%$ merchurochrome solution and quantified by microscopy. $\mathrm{IC}_{50}$ values were determined by logistic regression using Maximum Likelihood Estimation to find best fit ${ }^{41}$.

Gametogenesis assays. Plasmodium falciparum clone 3D7A (obtained from Dr Lisa Ranford-Cartwright, University of Edinburgh) gametocytes were initiated and cultured using an adapted version of the traditional Trager and Jenson method modified by Fivelman and colleagues ${ }^{42}$. Stage $\mathrm{V}$ gametocytes were purified on a $60 \%$ Percoll gradient. Purified parasite samples were diluted in warm complete media (RPMI-1640 (Sigma), 0.5\% Albumax (Invitrogen), 0.03\% L-glutamine (Sigma), $0.2 \%$ Glucose (Sigma), $1 \times$ hypoxanthine (Sigma)), to a volume that would allow a final volume of $100 \mu \mathrm{M}$ per sample and a haematocrit of $<10 \%$. Test compounds were added to $1.5 \mathrm{ml}$ tubes and $100 \mu \mathrm{l}$ of parasite suspension added to give a final concentration of $30 \mu \mathrm{M}$ xanthurenic acid, $2 \mu \mathrm{M}$ ML1, and $1 \mu \mathrm{M}$ ML10. Control tubes containing DMSO only were kept at $37^{\circ} \mathrm{C}$. All other samples were incubated at room temperature for $30 \mathrm{~min}$ immediately after addition of test compounds. Samples were then washed once in warm RPMI followed by removal of the supernatant to leave a final haematocrit of roughly $50 \%$. Samples were smeared on glass slides and fixed in 100\% methanol (VWR) then stained for 15 min in 10\% Giemsa R66 (VWR) and observed by light microscopy. The number of gametocytes at stage $\mathrm{V}$ and rounded up per 900 erythrocytes were counted three consecutive times to produce an average count. Numbers of parasites rounded up were compared to the no drug control.

Cytotoxicity assays with human cell lines. A549 (lung carcinoma; ATCC, Ref ATCC $^{\circledast}$ CCL- $185^{\circ \mathrm{m}}$ ), plated at $7.5 \times 10^{3}$ cells/well; HT- 29 (colorectal adenocarcinoma; ATCC, Ref. ATCC ${ }^{\circledast}$ HTB- $38^{m}$ ), plated at $7.5 \times 10^{3}$ cells/well; and MCF7 (breast adenocarcinoma; ATCC, Ref. ATCC ${ }^{\oplus}$ HTB- $22^{\mathrm{m}}$ ), plated at $9 \times 10^{3}$ cells $/$ well, were placed into clear bottom, opaque 96 well plates in cell line-specific medium and allowed to attach overnight. Cells were incubated with nine concentrations of ML10 (0.001-10 $\mu \mathrm{M}$ in 0.1\% DMSO) and grown in MEM Eagle medium (MEMEBSS with Non-Essential Amino Acids w/o L-Glutamine; Lonza BE12-662F), 10\% [v/v] FCS, 1× GlutaMAX ${ }^{\text {ma }}$ Supplement (Life Technologies, 35050061) and 50 units/ $\mathrm{ml}$ penicillin and $50 \mu \mathrm{g} / \mathrm{ml}$ streptomycin for $48 \mathrm{~h}$. Six independent assays were performed for each line and were carried out by CXR Biosciences Ltd. End point for cytotoxicity assessment was carried out using an ATP depletion kit (CellTiterGlo Luminescent Cell Viability Assay for ATP quantitation (Promega, Cat \# G7572)). Results expressed relative to the $0.1 \%$ DMSO control. Unpaired Student's $t$-tests were performed using GraphPad Prism software (7.0).

Crystallography. For crystallography, recombinant PvPKG was expressed and purified using a previously described baculovirus system ${ }^{43}$. Crystals were obtained by setting up protein samples in sitting drop vapor diffusion experiments at $18^{\circ} \mathrm{C}$. For ML1, the following crystallization conditions were used: 10\% PEG $5000 \mathrm{MME}$, $5 \%$ tacsimate, $0.1 \mathrm{M}$ HEPES pH 7.0, $15 \mathrm{mM}$ spermidine, and 25\% glycerol. The crystallization conditions for ML10 were: 15.5\% PEG 3350, 0.1 M HEPES pH 7, 0.1 M succinate $\mathrm{pH}$ 7.0. For both, data were collected at beam line 19ID of Argonne National Laboratory's Advanced Photon Source (http://www.sbc.anl.gov/index. $\mathrm{html}$ ) and processed using HKL- $3000^{44}$ (Table 2). The PvPKG-ML1 co-structure was solved using Phaser for molecular replacement and the previously deposited PvPKG-apo coordinates (PDB code: 5DYL) as a search model. Refinement was carried out using the Buster refinement software (version 2.10.0. developed by Bricogne et al., 2011 Cambridge, UK: Global Phasing Ltd. and REFMAC ${ }^{45}$ combined with iterative manual model building using the molecular graphics program $\mathrm{Coot}^{46}$ to a final R factor of $21.9 \%$. The PvPKG-ML10 co-structure was determined by refining the PvPKG apo structure (PDB code 5DYL) against the data acquired from the isomorphous complex crystals. The structure was refined using REFMAC $^{45}$ to a final R factors of $22.5 \%$. The geometry of the final models was checked using MolProbity ${ }^{47}$ for reasonable clash scores and no ramachandran outliers. Crystallographic details and refinement statistics are summarized in Table 2. The coordinates have been deposited in the Protein Data Bank with the PDB codes 5FET (ML1) and 5EZR (ML10). Stereo images of electron density maps for the compounds are shown in Supplementary Fig. 14.

Data availability. Data corresponding to crystal structures presented can be found at the RCSB Protein Data Bank (http://www.rcsb.org/pdb/home/home.do) with the following codes:

PvPKG apo: 5DYL, PvPKG with ML1: 5FET, PvPKG with ML10: 5EZR. All relevant data are available from the authors upon request.

Received: 20 February 2017 Accepted: 10 July 2017

Published online: 05 September 2017

\section{References}

1. WHO, World Malaria Report 2016 (World Health Organization: Geneva, 2016).

2. WHO, World Malaria Report 2014 (World Health Organisation: Geneva, 2014)

3. Amaratunga, C., et al., Dihydroartemisinin-piperaquine resistance in Plasmodium falciparum malaria in Cambodia: a multisite prospective cohort study. Lancet Infect. Dis., 16, 357-365 (2016).

4. Dondorp, A. M. et al. The threat of artemisinin-resistant malaria. N. Engl. J. Med. 365, 1073-1075 (2011).

5. Noedl, H. et al. Evidence of artemisinin-resistant malaria in western Cambodia N. Engl. J. Med. 359, 2619-2620 (2008).

6. Ashley, E. A. et al. Spread of artemisinin resistance in Plasmodium falciparum malaria. N. Engl. J. Med. 371, 411-423 (2014).

7. Sibley, C. H. Observing in real time the evolution of artemisinin resistance in Plasmodium falciparum. BMC Med. 13, 67 (2015).

8. Deng, W. et al. The role of two novel regulatory sites in the activation of the cGMP-dependent protein kinase from Plasmodium falciparum. Biochem. J. 374, 559-565 (2003).

9. Donald, R. G. et al. Anticoccidial kinase inhibitors: identification of protein kinase targets secondary to cGMP-dependent protein kinase. Mol. Biochem. Parasitol. 149, 86-98 (2006).

10. Gurnett, A. M. et al. Purification and molecular characterization of cGMPdependent protein kinase from Apicomplexan parasites. A novel chemotherapeutic target. J. Biol. Chem. 277, 15913-15922 (2002).

11. Huang, D. et al. Kinase selectivity potential for inhibitors targeting the ATP binding site: a network analysis. Bioinformatics. 26, 198-204 (2010).

12. Alam, M. M. et al. Phosphoproteomics reveals malaria parasite Protein Kinase $\mathrm{G}$ as a signalling hub regulating egress and invasion. Nat. Commun. 6, 7285 (2015). 
13. Brochet, M. et al. Phosphoinositide metabolism links cGMP-dependent protein kinase $\mathrm{G}$ to essential $\mathrm{Ca} 2+$ signals at key decision points in the life cycle of malaria parasites. PLoS Biol. 12, e1001806 (2014).

14. Diaz, C. A. et al. Characterization of Plasmodium falciparum cGMP-dependent protein kinase (PfPKG): antiparasitic activity of a PKG inhibitor. Mol. Biochem. Parasitol. 146, 78-88 (2006).

15. McRobert, L. et al. Gametogenesis in malaria parasites is mediated by the cGMP-dependent protein kinase. PLoS Biol. 6, el39 (2008).

16. Moon, R. W. et al. A cyclic GMP signalling module that regulates gliding motility in a malaria parasite. PLoS Pathog. 5, e1000599 (2009).

17. Taylor, H. M. et al. The malaria parasite cyclic GMP-dependent protein kinase plays a central role in blood-stage schizogony. Eukaryot. Cell 9, 37-45 (2010).

18. Govindasamy, K. et al. Invasion of hepatocytes by Plasmodium sporozoites requires cGMP-dependent protein kinase and calcium dependent protein kinase 4. Mol. Microbiol. 102, 349-363 (2016).

19. Collins, C. R. et al. Malaria parasite cGMP-dependent protein kinase regulates blood stage merozoite secretory organelle discharge and egress. PLoS Pathog. 9, e1003344 (2013)

20. Dvorin, J. D. et al. A plant-like kinase in Plasmodium falciparum regulates parasite egress from erythrocytes. Science 328, 910-912 (2010).

21. Peters, W. The chemotherapy of rodent malaria, XXII. The value of drugresistant strains of P. berghei in screening for blood schizontocidal activity. Ann. Trop. Med. Parasitol. 69, 155-171 (1975).

22. Jimenez-Diaz, M. B. et al. Improved murine model of malaria using Plasmodium falciparum competent strains and non-myelodepleted NOD-scid IL2Rgammanull mice engrafted with human erythrocytes. Antimicrob. Agents Chemother. 53, 4533-4536 (2009).

23. Tewari, R. et al. Development of a transgenic Plasmodium berghei line $(\mathrm{Pb}$ pfpkg) expressing the P. falciparum cGMP-dependent protein kinase, a novel antimalarial drug target. PLoS ONE 9, e96923 (2014).

24. Sanz, L. M. et al. P. falciparum in vitro killing rates allow to discriminate between different antimalarial mode-of-action. PLoS ONE 7, e30949 (2012).

25. Ponnudurai, T. et al. Infectivity of cultured Plasmodium falciparum gametocytes to mosquitoes. Parasitology 98, 165-173 (1989).

26. Eastman, R. T. et al. Piperaquine resistance is associated with a copy number variation on chromosome 5 in drug-pressured Plasmodium falciparum parasites. Antimicrob. Agents Chemother. 55, 3908-3916 (2011).

27. Baragana, B. et al. A novel multiple-stage antimalarial agent that inhibits protein synthesis. Nature 522, 315-320 (2015).

28. Jimenez-Diaz, M. B. et al. (+)-SJ733, a clinical candidate for malaria that acts through ATP4 to induce rapid host-mediated clearance of Plasmodium. Proc. Natl Acad. Sci. USA 111, E5455-E5462 (2014).

29. McNamara, C. W. et al. Targeting Plasmodium PI(4)K to eliminate malaria. Nature 504, 248-253 (2013).

30. Rottmann, M. et al. Spiroindolones, a potent compound class for the treatment of malaria. Science 329, 1175-1180 (2010).

31. Vaidya, A. B. et al. Pyrazoleamide compounds are potent antimalarials that target $\mathrm{Na}+$ homeostasis in intraerythrocytic Plasmodium falciparum. Nat. Commun. 5, 5521 (2014).

32. White, N. J. et al. Spiroindolone KAE609 for falciparum and vivax malaria. $N$. Engl. J. Med. 371, 403-410 (2014).

33. Gross, S. et al. Targeting cancer with kinase inhibitors. J. Clin. Invest. 125, 1780-1789 (2015).

34. Falae, A. et al. Role of Plasmodium berghei cGMP-dependent protein kinase in late liver stage development. J. Biol. Chem. 285, 3282-3288 (2010).

35. Cowman, A. F. et al. Malaria: Biology and Disease. Cell 167, 610-624 (2016).

36. Desjardins, R. E. et al. Quantitative assessment of antimalarial activity in vitro by a semiautomated microdilution technique. Antimicrob. Agents Chemother. 16, 710-718 (1979)

37. Angulo-Barturen, I. et al. A murine model of falciparum-malaria by in vivo selection of competent strains in non-myelodepleted mice engrafted with human erythrocytes. PLoS ONE 3, e2252 (2008).

38. Bolscher, J. M. et al. A combination of new screening assays for prioritization of transmission-blocking antimalarials reveals distinct dynamics of marketed and experimental drugs. J. Antimicrob. Chemother. 70, 1357-1366 (2015).

39. Feldmann, A. M. \& Ponnudurai, T. Selection of Anopheles stephensi for refractoriness and susceptibility to Plasmodium falciparum. Med. Vet. Entomol. 3, 41-52 (1989).

40. Ponnudurai, T. et al. Cultivation of fertile Plasmodium falciparum gametocytes in semi-automated systems. 1. Static cultures. Trans. R. Soc. Trop. Med. Hyg. 76, 812-818 (1982)

41. Vos, M. W. et al. A semi-automated luminescence based standard membrane feeding assay identifies novel small molecules that inhibit transmission of malaria parasites by mosquitoes. Sci. Rep. 5, 18704 (2015).

42. Fivelman, Q. L. et al. Improved synchronous production of Plasmodium falciparum gametocytes in vitro. Mol. Biochem. Parasitol. 154, 119-123 (2007).
43. Bezerra, G. A. et al. Structure of human dipeptidyl peptidase 10 (DPPY): a modulator of neuronal Kv4 channels. Sci. Rep. 5, 8769 (2015).

44. Minor, W. et al. HKL-3000: the integration of data reduction and structure solution--from diffraction images to an initial model in minutes. Acta Crystallogr. D. Biol. Crystallogr. 62, 859-866 (2006).

45. Murshudov, G. N., Vagin, A. A. \& Dodson, E. J. Refinement of macromolecular structures by the maximum-likelihood method. Acta Crystallogr. D. Biol. Crystallogr. 53, 240-255 (1997).

46. Emsley, P. \& Cowtan, K. Coot: model-building tools for molecular graphics. Acta Crystallogr. D. Biol. Crystallogr. 60, 2126-2132 (2004).

47. Davis, I. W. et al. MolProbity: all-atom contacts and structure validation for proteins and nucleic acids. Nucleic Acids Res. 35, W375-W383 (2007).

\section{Acknowledgements}

We gratefully acknowledge funding from the MRC for the DPFS grant (G10000779) to D.A.B., C.A.K., A.T.M., and C.L.C. and Wellcome grants (106240/Z/14/Z and 094752/Z/ $10 / Z$ ) to D.A.B. and support from MMV to cover the costs of outsourced assays. We wish to thank María Santos Martinez (GlaxoSmithKline) for assistance generating PK data in the GSK $P$. falciparum mouse model and for reviewing the GSK data. We are grateful to Mike Blackman, Tony Holder (The Francis Crick Institute), Christian Flueck, Avnish Patel, Laura Drought, Maria Penzo, and Rob Moon (LSHTM), Jeremy Burrows, Simon Campbell, Dennis Schmatz (MMV), and Rita Tewari (University of Nottingham) for helpful discussions.

\section{Author contributions}

D.A.B. conceived the study, was principle investigator on the MRC DPFS grant supporting the study, and wrote the paper. L.B.S. performed in vitro and in vivo experimental work. J.M.L. co-led and performed medicinal chemistry. P.W.B. performed in vitro experimental work, and helped to plan the work and write the paper. K.H.A. coordinated drug discovery experimental work and collated and analyzed data. M.B.J.-D performed the in vivo efficacy test in the P. falciparum mouse model. M.E.B. purified recombinant PvPKG and generated co-crystal structures. K.B. performed structure assisted compound design and helped analyze crystallography data. K.J.D. generated and analyzed the transmission blocking data. N.S.B. performed chemistry. P.J.C. helped with kinase assays development. D.W. initiated the medium throughput kinase assay. D.J.H. E.S.-H., and M.C.W. performed chemistry. E.M.W. generated gametogenesis inhibition data. J.T.D. and M.J.L. contributed to in vivo work. L.M.S. contributed speed of kill data F.-J.G. contributed speed of kill data and helped to write the paper. S.B.F. led the $P$. falciparum mouse model pharmacokinetic data. R.H. led crystallography, analyzed structural data, and helped write the manuscript. T.B. helped to generate the transmission blocking data. I.A.-B. led the in vivo efficacy work in the P. falciparum mouse model and helped to write the paper. A.T.M. advised on the medicinal chemistry programme. S. L.C. advised on in vivo work and helped to write the paper. W.E.G. served as drug discovery advisor for the study and helped to write the paper. C.A.K. was project manager for the study. S.A.O. led the medicinal chemistry programme and helped to write the paper.

\section{Additional information}

Supplementary Information accompanies this paper at doi:10.1038/s41467-017-00572-x.

Competing interests: The authors declare no competing financial interests.

Reprints and permission information is available online at http://npg.nature.com/ reprintsandpermissions/

Publisher's note: Springer Nature remains neutral with regard to jurisdictional claims in published maps and institutional affiliations.

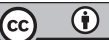

Open Access This article is licensed under a Creative Commons Attribution 4.0 International License, which permits use, sharing, adaptation, distribution and reproduction in any medium or format, as long as you give appropriate credit to the original author(s) and the source, provide a link to the Creative Commons license, and indicate if changes were made. The images or other third party material in this article are included in the article's Creative Commons license, unless indicated otherwise in a credit line to the material. If material is not included in the article's Creative Commons license and your intended use is not permitted by statutory regulation or exceeds the permitted use, you will need to obtain permission directly from the copyright holder. To view a copy of this license, visit http://creativecommons.org/ licenses/by/4.0/.

(c) The Author(s) 2017 